Article

\title{
Interleukin-4-Mediated Oxidative Stress Is Harmful to Hippocampal Neurons of Prothrombin Kringle-2-Lesioned Rat In Vivo
}

\author{
Young Cheul Chung ${ }^{1, \dagger}$, Jae Yeong Jeong ${ }^{2, \dagger}$ and Byung Kwan Jin $2,3, *$ \\ 1 Department of Predictive Toxicology, Korea Institute of Toxicology, Daejeon 34114, Korea; \\ youngcheul.chung@kitox.re.kr \\ 2 Department of Biochemistry \& Molecular Biology, School of Medicine, Kyung Hee University, Seoul 02447, \\ Korea; jyoung0229@khu.ac.kr \\ 3 Department of Neuroscience, Graduate School, Kyung Hee University, Seoul 02447, Korea \\ * Correspondence: bkjin@khu.ac.kr; Tel.: +82-2-961-9288; Fax: +82-2-969-4570 \\ + These authors contributed equally to this work.
}

Received: 5 October 2020; Accepted: 27 October 2020; Published: 30 October 2020

\begin{abstract}
The present study investigated the effects of reactive microglia/macrophages-derived interleukin-4 (IL-4) on hippocampal neurons in prothrombin kringle-2 (pKr-2)-lesioned rats. pKr-2 was unilaterally injected into hippocampus in the absence or presence of IL-4 neutralizing antibody (IL-4Nab). Immunohistochemical analysis showed a significant loss of $\mathrm{Niss}^{+}$and $\mathrm{NeuN}^{+}$cells and activation of microglia/macrophages (increase in reactive OX- $42^{+}$and $\mathrm{OX}-6^{+}$cells) in the hippocampus at 7 days after $\mathrm{pKr}-2$ injection. The levels of IL-4 expression were upregulated in the reactive OX- $42^{+}$microglia/macrophages as early as 1 day, maximal at 3 days and maintained up to 7 days after $\mathrm{pKr}-2$ injection. Treatment with IL-4Nab significantly increased neuronal survival in pKr-2-treated CA1 layer of hippocampus in vivo. Accompanying neuroprotection, IL-4 neutralization inhibited activation of microglia/macrophages, reactive oxygen species-derived oxidative damages, production of myeloperoxidase- and inducible nitric oxide synthase-derived reactive nitrogen species and nitrosative damages as analyzed by immunohistochemistry and hydroethidine histochemistry. These results suggest that endogenous IL-4 expressed on reactive microglia/macrophages mediates oxidative/nitrosative stress and play a critical role on neurodegeneration of hippocampal CA1 layer in vivo.
\end{abstract}

Keywords: oxidative/nitrosative stress; neurodegeneration; microglia/macrophages; prothrombin-kringle-2; interkeukin-4

\section{Introduction}

Oxidative/nitrosative stress, defined as 'an imbalance in pro-oxidants and antioxidants with associated disruption of redox circuitry and macromolecular damage' is strongly associated with the development of neuronal death and dysfunction [1,2]. The balance between reactive oxygen species/reactive nitrogen species (ROS/RNS) production and the antioxidant defense is modulated by multiple sources such as ROS/RNS production enzymes and free radical scavengers to maintain cellular functions $[1,3]$. Accumulating evidence suggests that oxidative/nitrosative stress is well-known feature of neurodegenerative disorders, including Alzheimer's disease (AD), Parkinson's disease (PD) and amyotrophic lateral sclerosis (ALS) $[1,4,5]$. Accordingly, antioxidants may be beneficial for the treatment of neurodegenerative disorders, that are associated with oxidative damage.

Microglia, resident macrophages in the brain, are known to neuronal support cells that maintain synaptic remodeling and brain homeostasis in healthy conditions [6,7]. Under pathological conditions, 
microglia are transformed to become reactive and then to determine whether a neuron should be cleared (neurotoxic) or salvageable (neurotrophic) $[7,8]$. In a neurodegenerative condition, reactive microglia preserve a neurotoxic state through triggering production of inflammatory molecules and oxidative/nitrosative stress [4,6-8].

Prothrombin kringle-2 (pKr-2), the second domains of prothrombin which is generated by active thrombin, is known to directly induce microglial activation and produce neurotoxic molecules such as inducible nitric oxide synthase (iNOS)-derived ROS/RNS, tumor necrosis factor-alpha (TNF- $\alpha$ ) and interleukin-1beta (IL-1 $\beta$ ) in vivo and in vitro [9-11]. pKr-2-induced microglial activation and neurotoxic molecules contribute to neurodegeneration in the cortex and nigrostriatal dopamine system in vivo $[9,10,12]$. In addition, up-regulated levels of $\mathrm{pKr}-2$ and thrombin were observed in the postmortem brains of $\mathrm{AD}$ and $\mathrm{PD}$ patients, indicating the relevance of $\mathrm{pKr}-2$ in the neurodegenerative disorders [12,13].

Interleukin-4 (IL-4) is well-known anti-inflammatory cytokine and has diverse immunomodulatory functions on cell repair and regeneration in the central nervous system [14,15]. In the context of neuroinflammation under pathological conditions, it exerts either harmful or beneficial effects on neurons by regulating oxidative stress in vivo and in vitro $[9,11,15-17]$. Thus, the present study examined whether $\mathrm{pKr}$-2-induced neurotoxicity could contribute to endogenous production of IL-4, which is neurotoxic or neuroprotective in hippocampus in vivo.

\section{Materials and Methods}

\subsection{Chemicals}

Prothrombin kringle-2 (pKr-2) was purchased from Haematologic Technologies (Essex Junction, VT, USA). Interleukin-4 neutralizing antibody (IL-4Nab) and non-specific IgG were obtained from R\&D system (Minneapolis, MN, USA), and dihydroethidium (hydroethidine) was purchased from ThermoFisher Scientific (Waltham, MA, USA).

\subsection{Animals}

Animal experiments were carried out according to the guideline set by Committee on Animal Research of Kyung Hee University (KHUASP-20-235, 30 June 2020). Female Sprague-Dawley rats were used in this study. They were housed three per cage under a $12 \mathrm{~h}$ light/dark cycle in a temperature-controlled room $\left(21-23^{\circ} \mathrm{C}\right)$. Throughout the experiment, food and water were available ad libitum. All efforts were made to minimize animal suffering and the minimal number of animals necessary to produce reliable scientific data were used.

\subsection{Intrahippocampal Microinjection}

As described in References [9,18], rats weighing 240-260 g (10 weeks of age) were anesthetized with intraperitoneally (i.p.) administered chloral hydrate (360 mg/kg; Sigma-Aldrich., St. Louis, MO, USA) and placed in a stereotaxic apparatus. The pKr-2 (48 $\mu \mathrm{g}$ in $4 \mu \mathrm{L}$ phosphate-buffered saline (PBS; Gibco., Paisley, UK)) was unilaterally injected into right CA1 layer of hippocampus (3.6 mm posterior to bregma, $2.0 \mathrm{~mm}$ lateral to the midline, $2.8 \mathrm{~mm}$ beneath the skull of the brain according to the atlas of Paxinos and Watson [19] with 30 gauge of Hamilton syringe at a rate of $0.2 \mu \mathrm{L}$ per minute. For neutralization of interleukin-4 (IL-4) $[9,18]$, the mixture of pKr-2 and IL-4Nab $(1 \mu \mathrm{g} / \mu \mathrm{L})$ or non-specific IgG as a control $(1 \mu \mathrm{g} / \mu \mathrm{L})$ was injected into the equivalent coordinate of right CA1 layer of hippocampus.

\subsection{Immunohistochemistry (IHC) and Immunofluorescence (IF) Staining}

Animals were deeply anesthetized with an overdose of chloral hydrate (intraperitoneally) and perfused transcardially with physiological saline containing $0.5 \%$ sodium nitrate and heparin $(10 \mathrm{U} / \mathrm{mL})$, followed by $4 \%$ paraformaldehyde in $0.1 \mathrm{M}$ phosphate buffer ( $\mathrm{pH} 7.4$ ). Brains were then removed, 
post-fixed overnight and transferred to $30 \%$ sucrose solution. Serial coronal sections were cut sectioned at $40 \mu \mathrm{m}$ thickness on a sliding microtome. Every sixth section was processed for immunohistochemical staining as described $[9,20]$. In brief, free-floating brain sections were rinsed with PBS, quenched in PBS containing 3\% $\mathrm{H}_{2} \mathrm{O}_{2}$, blocked in PBS containing 1\% bovine serum albumin (BSA; Millipore corporation., Kankakee, IL, USA) with $0.2 \%$ triton X-100 (Sigma-Aldrich), and then incubated overnight at room temperature with primary antibodies (Table 1). The following day, brain sections were rinsed in PBS containing 0.5\% BSA, incubated with the biotin-conjugated mouse IgG (Table 2), processed with an avidin-biotin complex kit (Vector Laboratories., Burlingame, CA, USA) and visualized 0.05\% 3,3'-diaminobenzidine tetrahydrochloride hydrate (DAB; Sigma-Aldrich) and 0.003\% hydrogen peroxide. Labeled tissue sections were then mounted on adhesive microscope slides and viewed under a bright-field microscope (Olympus BX51; Olympus Optical., Tokyo, Japan). The primary antibodies used were as follows: mouse anti-OX-42 for microglia/macrophages and neutrophils, mouse anti-OX-6 for activated microglia, mouse anti-neuron-specific nuclear protein (NeuN) for neurons and mouse anti-8-hydroxy-2-deoxy guanosine (8-OHdG) for detecting oxidative DNA damage (Table 1).

Table 1. Primary antibodies used for immunohistochemistry (IHC) and immunofluorescence (IF).

\begin{tabular}{cccc}
\hline Primary Antibody & Dilution & Company & Catalog No. \\
\hline OX-42 & $1: 400$ & Bio-rad & MCA275G \\
OX-6 & $1: 400$ & BD Biosciences & 554926 \\
FITC-TL & $1: 1000$ & Vector Laboratories & FL-1171 \\
NeuN & $1: 1000$ & Merck & MAB377 \\
GFAP & $1: 500$ & Sigma-Aldrich & G3893 \\
IL-4 & $1: 400$ & Abbiotec & 251223 \\
MPO & $1: 500$ & DakoCytomation & A0398 \\
iNOS & $1: 200$ & BD Biosciences & 610333 \\
8-OHdG & $1: 300$ & Jaica & MOG-100P \\
Nitrotyrosine & $1: 50$ & Abcam & ab7048 \\
\hline
\end{tabular}

Table 2. Secondary antibodies used for IHC and IF.

\begin{tabular}{cccc}
\hline Secondary Antibody & Dilution & Company & Catalog No. \\
\hline Biotin-conjugated anti-mouse IgG & $1: 400$ & KPL & $16-18-15$ \\
FITC-conjugated anti-mouse IgG & $1: 500$ & Sigma-Aldrich & AP124F \\
Fluorescein-conjugated anti-mouse IgG & $1: 300$ & Vector Laboratories & FI-2000 \\
Cy3-conjugated anti-rabbit IgG & $1: 1000$ & Sigma-Aldrich & AP132C \\
\hline
\end{tabular}

For Nissl staining, some of the hippocampal tissues were mounted on adhesive microscope slides, dried, stained in $0.5 \%$ cresyl violet acetate solution (Sigma-Aldrich), dehydrated, covered, and then analyzed under a bright-field microscope.

For immunofluorescence staining, tissue sections were processed as described [9,20]. Free-floating sections were mounted on adhesive microscope slides and dried at room temperature. After washing, sections were incubated with primary antibodies overnight at $4{ }^{\circ} \mathrm{C}$ (Table 1). The following day, brain sections were rinsed in PBS containing $0.5 \%$ BSA, incubated with the appropriate fluorescence-conjugated secondary antibodies. The sections were washed, covered with vectashield mounting medium for fluorescence with DAPI (Vector Laboratories), and analyzed under confocal microscope (LSM700; Carl Zeiss., Oberkochen, Germany). The primary antibodies used were as follows: rabbit anti-IL-4, fluorescein isothiocyanate (FITC)-labeled tomato lectin (TL) and mouse anti-OX-42 for microglia/macrophages and neutrophils, NeuN, mouse anti-glial fibrillary acidic protein (GFAP) for astrocytes, rabbit anti-myeloperoxidase (MPO), rabbit anti-inducible nitric oxide (iNOS), and mouse anti-nitrotyrosine for recognizing ROS/RNS-dependent protein damage (Table 1). The fluorescence-conjugated secondary antibodies used were as follows: FITC-conjugated anti-mouse, fluorescein-conjugated anti mouse, and Cy3-conjugated anti-rabbit (Table 2). 


\subsection{In Situ Detection of $\mathrm{O}_{2}{ }^{-}$and $\mathrm{O}_{2}{ }^{-}$-Derived Oxidants}

In situ $\mathrm{O}_{2}{ }^{-}$production was visualized by hydroethidine histochemistry $[9,20]$. At 3 days after pKr-2 injection, rats received intravenous injection of hydroethidine $(1 \mathrm{mg} / \mathrm{kg}$ in PBS containing $1 \%$ dimethyl sulfoxide) into tail vein. After $45 \mathrm{~min}$, rats were transcardially perfused and fixed. Brain tissues were cut sectioned at $40 \mu \mathrm{m}$ thickness on a sliding microtome and mounted on adhesive microscope slides. The oxidized hydroethidine product, ethidium, was investigated by confocal microscopy (LSM700) and merged with DAPI solution (Vector Laboratories).

\subsection{Quantification of Neurons in Hippocampal CA1 Layer}

To quantify the number of hippocampal neurons in CA1 layer of hippocampus, neurons were counted on four levels of the dorsal hippocampus, respectively. Specifically, alternate sections were obtained from the following coordinates: $3.3,3.6,4.16$, and $4.3 \mathrm{~mm}$ posterior to bregma (totally, 4 areas for each animal) were used to count cells in the CA1 layer. Using a bright-field microscope at a magnification of 40X, the number of neurons in the CA1 layer was counted and quantified as the number of CA1 per millimeter of linear length (Hippocampal CA1 neurons/mm), as described previously $[18,21]$. To maintain consistency between each animal, we counted the number of neurons from $1.5 \mathrm{~mm}$ lateral to the midline. Only neurons with normal visible nuclei were counted. The mean number of CA1 neurons per millimeter in 4 areas for each animal was calculated for each experimental group.

\subsection{Image J Analysis}

To analyze and quantify the expression of IL-4, 8-OHdG, MPO, iNOS, nitrotyrosine and oxidized hydroethidine product in pKr-2-injected CA1 layer of hippocampus with absence or presence of IL-4Nab, images were obtained from the same area in each tissue sample. Imaging data were analyzed as pixel value, the number of pixels above the threshold, through Image J (National Institutes of Health). Image J was used to quantify chromogenic signal intensity of immunofluorescence on image. All original images were collected, converted to 8-bit grayscale and the minimum threshold values of images were adjusted at the endpoint of histogram for excluding the background signal. Then, the signals were quantified as pixel values and normalized by unstained area.

\subsection{Statistical Analysis}

Results are expressed as means \pm SEM. For statistical evaluation, the data was assessed by one-way ANOVA Newman-Keuls analyses and Student's unpaired $t$-test (GraphPad Software, San Diego, CA, USA). Significance level was set at $p<0.05$.

\section{Results}

\section{1. $p K r-2$ Induces Activation of Microglia/Macrophages and Neuronal Death in the Hippocampus In Vivo}

Recent findings, including ours, indicate that $\mathrm{pKr}-2$ activates microglia in the cerebral cortex and substantia nigra in vivo $[9,11]$. The present study determined the activation of microglia/macrophages by $\mathrm{pKr}-2$ in the CA1 layer of hippocampal neurons in vivo. To test this, PBS as a control or $\mathrm{pKr}-2$ $(48 \mu \mathrm{g} / 4 \mu \mathrm{L})$ was unilaterally injected into the CA1 layer of rat hippocampus. Immunohistochemical analysis demonstrated that $\mathrm{OX}-42$-immunopositive $\left(\mathrm{OX}-42^{+}\right)$microglia/macrophages exhibited a resting state, small cell bodies with ramified processes in the PBS-injected CA1 layer at 7 days after injection (Figure $1 \mathrm{~A}, \mathrm{~B})$. In contrast, the majority of $\mathrm{OX}-42^{+}$microglia/macrophages displayed an activated state, large cell bodies with short, thick, or no processes, which were observed as early as 1 day after $\mathrm{pKr}-2$ injection (Figure $1 \mathrm{C}, \mathrm{D}$ ) and sustained up to 7 days after $\mathrm{pKr}-2$ injection (Figure 1E-H). Similar to changes of OX-42+ cells, many OX $-6^{+}$cells were observed in the CA1 layer of hippocampus as early as 1 day after $\mathrm{pKr}-2$ injection (Figure $1 \mathrm{~K}, \mathrm{~L}, \mathrm{Q}$ ) and maintained up to 7 days after pKr-2 injection (Figure 1M-Q), whereas in PBS-injected control, few of OX-6 $6^{+}$cells were seen (Figure 1I,J,Q). 

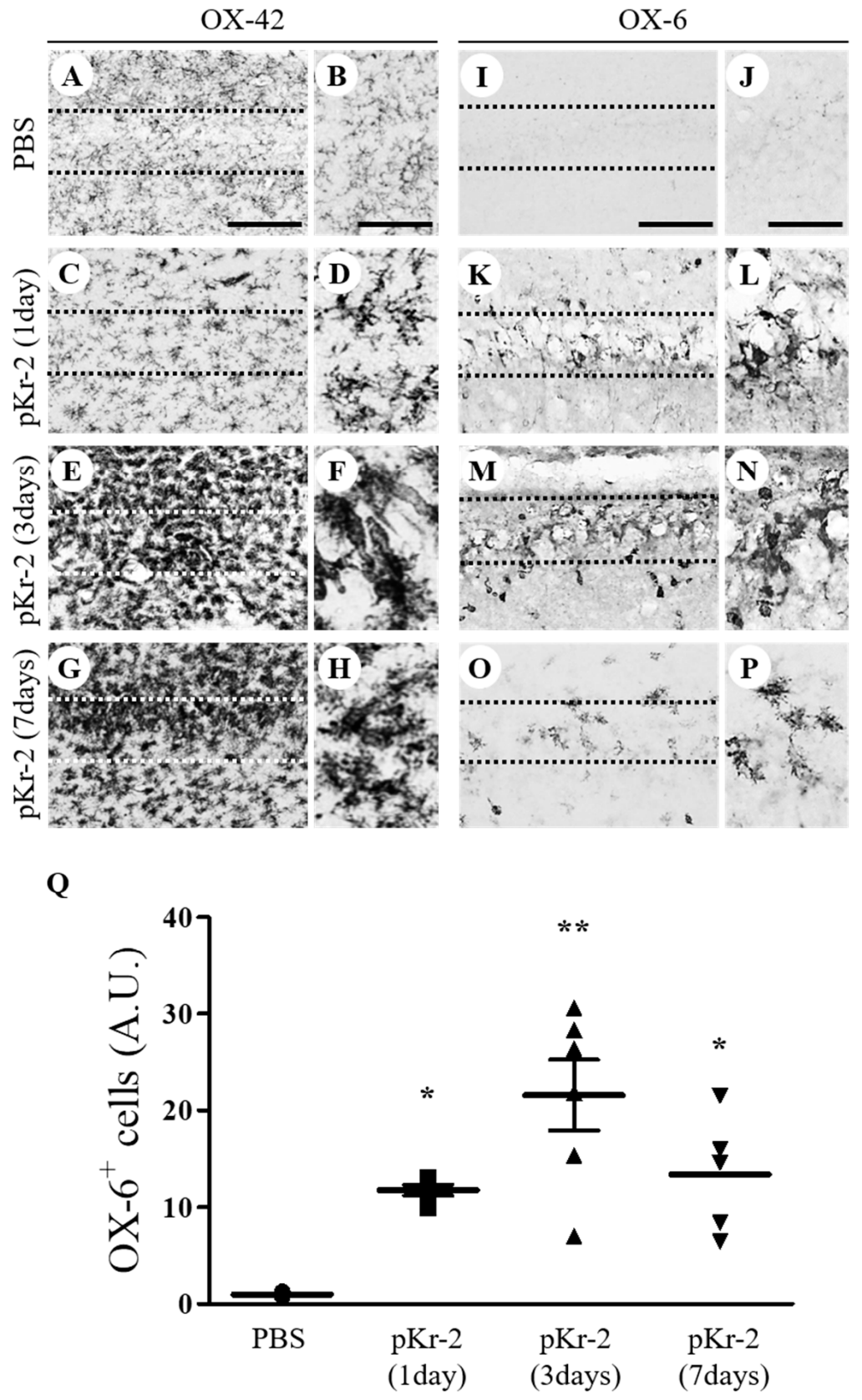

Figure 1. Prothrombin kringle-2-induced microglial/macrophages activation in the CA1 area of the hippocampus in vivo. Phosphate-buffered saline (PBS) (A,B,I,J) as a control or prothrombin kringle-2 (pKr-2; C-H, K-P; $48 \mu \mathrm{g} / 4 \mu \mathrm{L}$ ) was unilaterally injected into the CA1 layer of rat hippocampus. Animals were transcardially perfused at indicated time points and brain tissues were prepared for immunohistochemical staining. Every sixth serial sections were selected and immunostained with OX-42 (complement receptor 3, CR3; A-H) to identify microglia/macrophages and neutrophils or OX-6 (major histocompatibility complex class II; I-P) to identify activated microglia at 1 day $(\mathbf{C}, \mathbf{D}, \mathbf{K}, \mathbf{L}), 3$ days $(\mathbf{E}, \mathbf{F}, \mathbf{M}, \mathbf{N})$ and 7 days $(\mathbf{G}, \mathbf{H}, \mathbf{O}, \mathbf{P})$ after intrahippocampal injection of $\mathrm{pKr}-2$. (B,D,F,H,J,L,N and $\mathbf{P})$ show higher magnifications of (A,C,E,G,I,K,M and $\mathbf{O})$, respectively. Scale bars, $100 \mu \mathrm{m}(\mathbf{A}, \mathbf{C}, \mathbf{E}, \mathbf{G}, \mathbf{I}, \mathbf{K}, \mathbf{M}$ and O), $100 \mu \mathrm{m}(\mathbf{B}, \mathbf{D}, \mathbf{F}$ and $\mathbf{H})$ and $50 \mu \mathrm{m}(\mathbf{J}, \mathbf{L}, \mathbf{N}$ and $\mathbf{P})$. (Q) Quantification of OX-6 ${ }^{+}$cells in the CA1 layer of pKr-2-injected hippocampus. ${ }^{*} p<0.01,{ }^{* *} p<0.001$ significantly different from PBS. mean $\pm \mathrm{SEM}$; $n=5$ to 7 in each group, ANOVA and Newman-Keuls analysis. Note the morphological changes from resting (small cell bodies with ramified processes) to activated state (large cell bodies with short processes) in the pKr-2-injected CA1 layer of hippocampus, compared with the PBS-injected control. 
As pKr-2 induces neurodegeneration in the cerebral cortex and substantia nigra [9-11], we hypothesized that $\mathrm{pKr}-2$ could produce neurodegeneration in the CA1 layer of the hippocampus. Sections adjacent to those used for Figure 1 were processed for Nissl staining and immunostaining for neuronal nuclei (NeuN) to detect general hippocampal neurons. Compared to PBS-injected control (Figure 2A,B), a significant reduction of Nissl-stained cells was induced as early as 1 day after pKr-2 injection (Figure 2C,D), increased at 3 days after pKr-2 injection (Figure 2E,F) and maximal showing marked loss of Nissl substances with severe gliosis at 7 days after pKr-2 injection (Figure 2G,H). NeuN immunohistochemical staining showed that a significant loss of $\mathrm{NeuN}^{+}$cells was seen in the CA1 layer of hippocampus as early as 1 day after $\mathrm{pKr}-2$ injection (Figure $2 \mathrm{~K}, \mathrm{~L}, \mathrm{Q}$ ), increased at 3 days after pKr-2 injection (Figure 2M-Q) and maximal at 7 days after $\mathrm{pKr}-2$ injection (Figure 2O,P,Q) compared to PBS-injected control (Figure 2I,J,Q). Taken together, these results indicate that pKr-2 induces activation microglia/macrophages as well as neurodegeneration in the CA1 layer of hippocampus in vivo in a time-dependent manner.
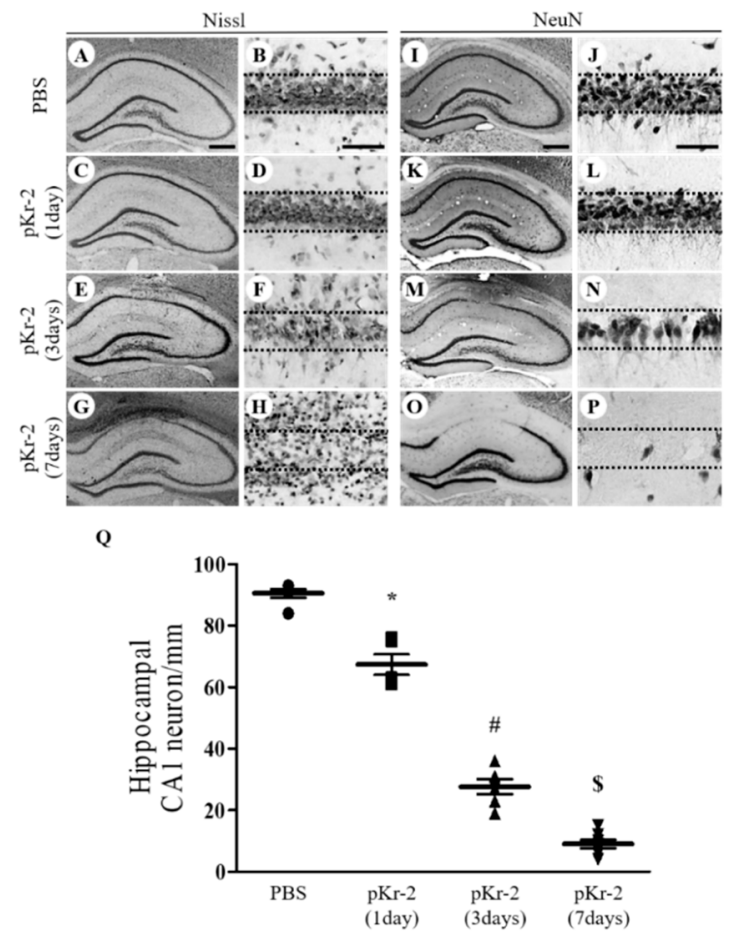

Figure 2. pKr-2-induced neurotoxicity in the CA1 layer of the hippocampus in vivo. Sections $(\mathbf{A}, \mathbf{B}, \mathbf{I}, \mathbf{J}$, PBS; C-H, K-P, pKr-2) adjacent to those used in Figure 1 were processed for Nissl staining or neuronal nuclei (NeuN) immunostaining at indicated time points. (B,D, $, \mathbf{F}, \mathbf{H}, \mathbf{J}, \mathbf{L}, \mathbf{N}$ and $\mathbf{P})$ show higher magnifications of (A,C,E,G,I,K,M and $\mathbf{O}$ ), respectively. (A-H) CA1 layer of the hippocampus stained for Nissl substance (cresyl violet). Scale bars, $500 \mu \mathrm{m}$ (A,C,E and G), $50 \mu \mathrm{m}$ (B,D,F and H). (I-P) NeuN immunostaining in the CA1 layer of hippocampus. Scale bars, $500 \mu \mathrm{m}(\mathbf{I}, \mathbf{K}, \mathbf{M}$ and $\mathbf{O}), 50 \mu \mathrm{m}$ $(\mathbf{J}, \mathbf{L}, \mathbf{N}$ and $\mathbf{P})$. (Q) Quantification of $\mathrm{NeuN}^{+}$cells in the CA1 layer of pKr-2-injected hippocampus. ${ }^{*} p<0.001$, significantly different from PBS. ${ }^{\#} p<0.001$, significantly different from pKr-2 (1day). $\$ p<0.001$, significantly different from $\mathrm{pKr}-2$ (3days). mean $\pm \mathrm{SEM} ; n=5$ to 7 in each group, ANOVA and Newman-Keuls analysis.

3.2. Endogenous IL-4 Expressed within Reactive Microglia/Macrophages Contributes to Neurodegeneration in pKr-2-Injected CA1 Layer of Hippocampus In Vivo

IL-4 expressed in CD11 $\mathrm{b}^{+}$-reactive microglia/macrophages contributes to degeneration of neurons in the $\mathrm{pKr}$-2-treated cortex in vivo [9] and in the thrombin- or beta-amyloid ${ }_{1-42}$-treated hippocampus in vivo [18,22]. Accordingly, we next examined whether $\mathrm{pKr}-2$ could induce expression of IL-4 within reactive microglia/macrophages in the CA1 layer of hippocampus, resulting in neurodegeneration. 
Immunohistochemical analysis demonstrated that endogenous expression of IL-4 was observed as early as 1 day after pKr-2 injection (Figure 3B,E), maximal at 3 days after pKr-2 injection (Figure 3C,E) and sustained up to 7 days after pKr-2 injection (Figure 3D,E) compared to PBS-injected control (Figure 3A,E).
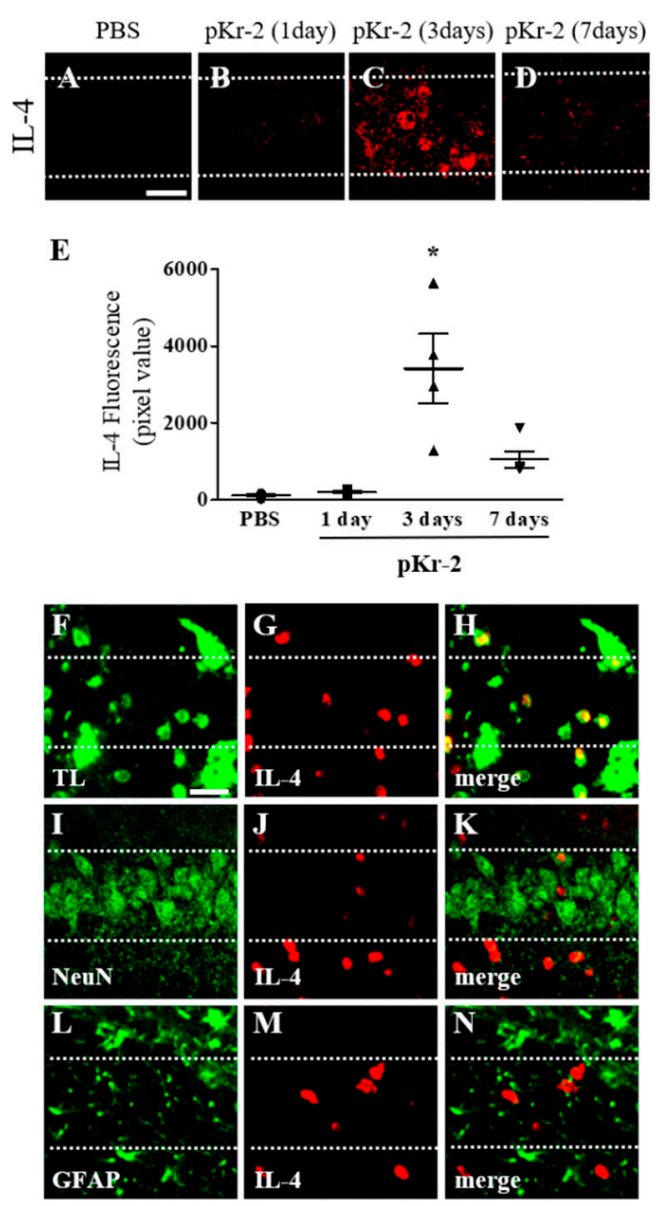

Figure 3. Expression of endogenous interleukin-4 in the CA1 layer of pKr-2-lesioned hippocampus in vivo. PBS (A) or pKr-2 (B-D, F-N) was unilaterally injected into the CA1 layer of rat hippocampus. Animals were transcardially perfused at various time points and brain sections were processed for immunohistochemical staining. (A-E) Immunofluorescence images of IL-4 (A-D) and quantification (E) in the CA1 layer of rat hippocampus at the indicated time points. ${ }^{*} p<0.001$, significantly different from PBS (control). mean \pm SEM; E, $n=4$ to 5 in each group, ANOVA and Newman-Keuls analysis. Scale bar, $40 \mu \mathrm{m}$. (F-N) Double immunofluorescence images of tomato lectin (TL; F, green) for microglia/macrophages and IL-4 (G, red) or neuronal nuclei (NeuN; I, green) for neurons and IL-4 (J, red) or glial fibrillary acidic protein (GFAP; L, green) for astrocytes and IL-4 (M, red) and both images are merged (yellow; $\mathbf{H}, \mathbf{K}$ and $\mathbf{N}$ ) in the CA1 layer of hippocampus at 3 days after $\mathrm{pKr}-2$ injection. Scale bar, $25 \mu \mathrm{m}$. These data are representative of four to five animals per group. Dotted lines indicate the CA1 layer of the hippocampus.

Expression of IL-4 was analyzed in tomato lectin (TL) ${ }^{+}$microglia/macrophages, $\mathrm{NeuN}^{+}$neurons and $\mathrm{GFAP}^{+}$astrocytes at 3 days after $\mathrm{pKr}-2$ injection. Expression of IL-4 was mainly localized in $\mathrm{TL}^{+}$microglia/macrophages (Figure $3 \mathrm{~F}-\mathrm{H}$ ), but neither in $\mathrm{NeuN}^{+}$neurons (Figure 3I-K) nor GFAP ${ }^{+}$ astrocytes (Figure 3L-N) in CA1 layer of hippocampus in vivo.

Next, IL-4-mediated neurotoxicity was assessed in CA1 layer of hippocampus in vivo at 3 days after $\mathrm{pKr}-2$ injection. Immunohistochemical analysis exhibited the significant loss of $\mathrm{Nissl}^{+}$and $\mathrm{NeuN}^{+}$ (Figure 4C,D,K) cells in pKr-2-injected CA1 layer of hippocampus compared to PBS-injected control 
(Figure 4A,B,K), respectively. To test IL-4-mediated neurodegeneration, IL-4 neutralizing antibody (IL-4Nab) was unilaterally co-injected with pKr-2 to block the action of IL-4 into the CA1 layer of rat hippocampus. Immunohistochemical analysis showed that treatment of IL-4Nab significantly prevented pKr-2-induced degeneration of $\mathrm{Nissl}^{+}$and $\mathrm{NeuN}^{+}$(Figure 4E,F,K) cells in the CA1 layer of the hippocampus. Treatment with IL-4Nab alone did not affect neuronal survival (Figure 4K). As a control, non-specific goat IgG with (Figure 5G,H,K) or without pKr-2 (Figure 5I-K) had little effect, similar to that observed with injection of $\mathrm{pKr}-2$ only.

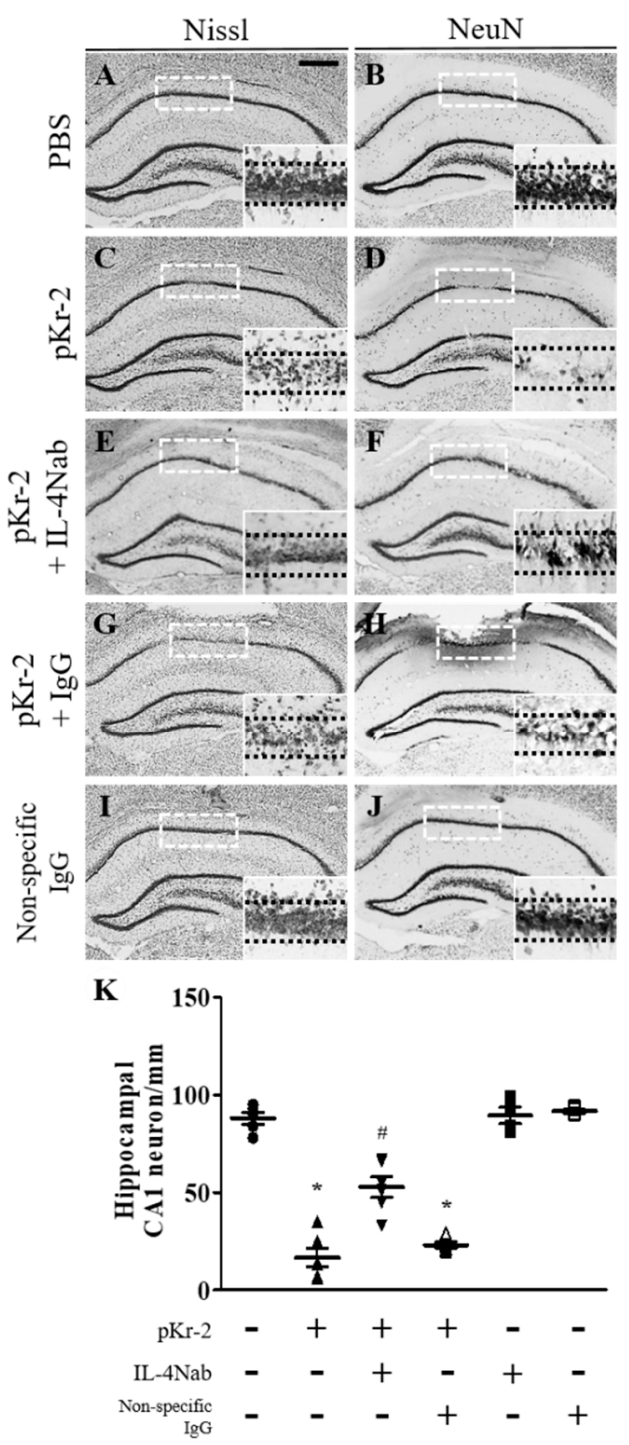

Figure 4. IL-4-mediated neurotoxicity in the CA1 layer of pKr-2-injected hippocampus in vivo. Animals unilaterally received intrahippocampal injection of PBS (A,B) as a control, pKr-2 (C,D), pKr-2 + IL-4Nab $(1 \mu \mathrm{g} ; \mathbf{E}, \mathbf{F}), \mathrm{pKr}-2+$ non-specific IgG $(1 \mu \mathrm{g} ; \mathbf{G}, \mathbf{H})$, and non-specific IgG only $(1 \mu \mathrm{g} ; \mathbf{I} \mathbf{I})$ ). At 3 days after injection, animals were transcardially perfused and brain tissues were processed for Nissl staining or neuronal nuclei $(\mathrm{NeuN})$ immunostaining at 3 days after $\mathrm{pKr}-2$ injection. (A,C,E,G,I) CA1 layer of the hippocampus stained for Nissl substance. (B,D,F,H,J) NeuN immunostaining in the CA1 layer of hippocampus. Insets show magnified photomicrographs in the CA1 layer marked by dotted rectangles, respectively. Scale bar, $500 \mu \mathrm{m}$. (K) Number of NeuN ${ }^{+}$cells in the CA1 layer of pKr-2-injected hippocampus in the absence or presence of IL-4Nab. ${ }^{*} p<0.001$, significantly different from PBS. ${ }^{\#} p<0.001$, significantly different from $\mathrm{pKr}-2$. mean $\pm \mathrm{SEM} ; n=4$ to 6 in each group, ANOVA and Newman-Keuls analysis. 


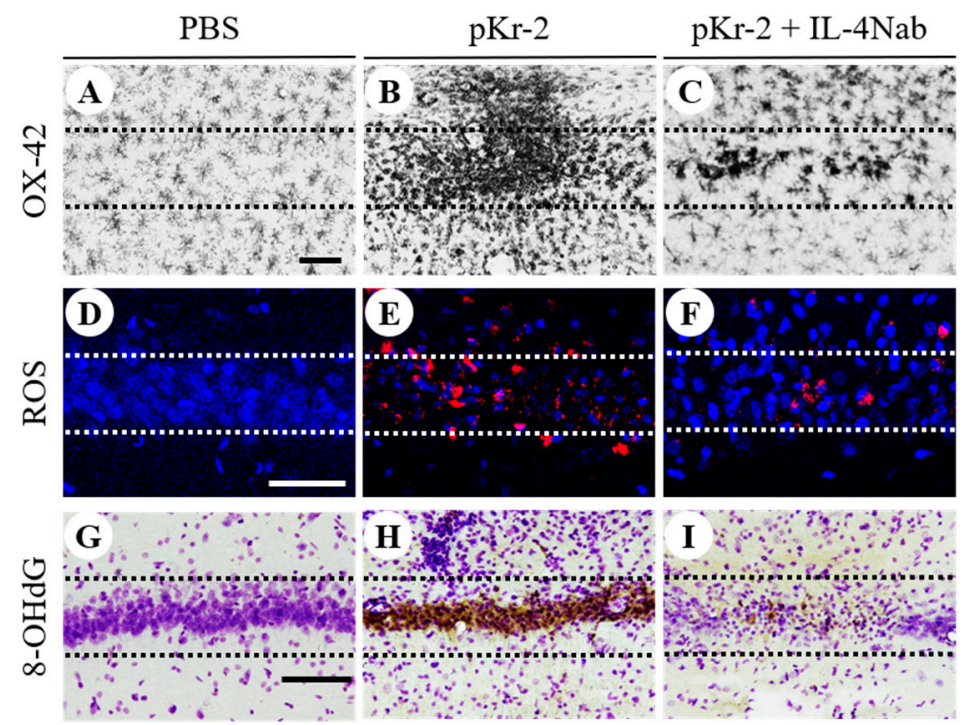

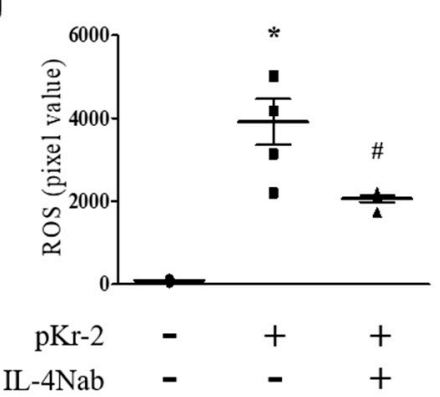

K

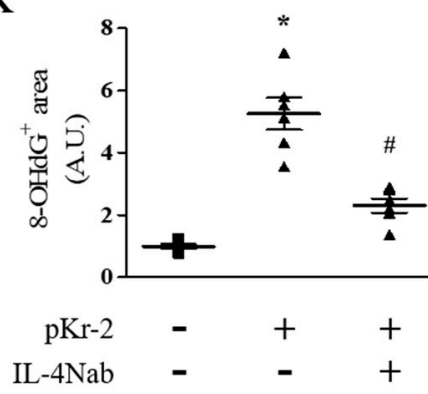

Figure 5. IL-4 induces activation of microglia/macrophages and oxidative stress in the CA1 layer of pKr-2-injected hippocampus in vivo. Sections (A,D,G (PBS); B,E,H (pKr-2); C,F,I (pKr-2 + IL-4Nab)) adjacent to those used in Figure 4 were processed for immunohistochemical staining or hydroethidine histochemistry. (A-C) CA1 layer of the hippocampus stained for OX-42 antibody to identify microglia/macrophages. Scale bar, $100 \mu \mathrm{m}$. (D-F) Hydroethidine histochemistry to detect oxidant production (ethidium fluorescence, red) in the CA1 layers of hippocampus. Nuclei were counterstained with DAPI (blue). Scale bar, $50 \mu \mathrm{m}$. (G-I) CA1 layers of the hippocampus stained for 8-hydroxy-2-deoxy guanosine (8-OHdG) antibody to detect oxidative DNA damages and counterstained with Nissl. Scale bar, $100 \mu \mathrm{m}$. (J) Quantification of ROS expression. ${ }^{*} p<0.001$, significantly different from PBS (control). ${ }^{\#} p<0.001$, significantly different from $\mathrm{pKr}-2$. mean $\pm \mathrm{SEM} ; n=4$ to 5 in each group, ANOVA and Newman-Keuls analysis. (K) Quantification of 8-OHdG expression. ${ }^{*} p<0.001$, significantly different from PBS. ${ }^{*} p<0.001$, significantly different from $\mathrm{pKr}-2$. mean \pm SEM; $n=4$ to 5 in each group, ANOVA and Newman-Keuls analysis. Dotted lines indicate the CA1 layer of the hippocampus.

3.3. IL-4 Induces Activation of Microglial/Macrophages, ROS Production and Oxidative Damages in pKr-2-Injected CA1 Layer of Hippocampus In Vivo

We previously reported that IL-4 regulates activation of microglia/macrophages in the pKr-2-treated cortex and lipopolysaccharide (LPS)-treated substantia nigra in vivo [9,23]. Accordingly, we examined whether IL-4 could activate microglia/macrophages in the CA1 layer of hippocampus in vivo at 3 days after $\mathrm{pKr}-2$ injection. Immunohistochemical analysis demonstrated that the majority of $\mathrm{OX}-42^{+}$ microglia/macrophages were activated in pKr-2-injected CA1 layer of hippocampus (Figure 5B) compared to PBS-injected hippocampus (Figure 5A). Treatment of IL-4Nab prevented pKr-2-induced activation of $\mathrm{OX}-42^{+}$microglia/macrophages in the CA1 layer of hippocampus (Figure 5C).

As IL-4 mediates $\mathrm{O}_{2}{ }^{-}$production in the thrombin- and beta-amyloid ${ }_{1-42}$-lesioned hippocampus or pKr-2-lesioned cortex $[9,18,22]$, we hypothesized that IL-4 could contribute to $\mathrm{O}_{2}{ }^{-}$production in pKr-2-treated hippocampus in vivo. To test this, hydroethidine histochemistry was performed for in 
situ visualization of $\mathrm{O}_{2}{ }^{-}$production. The fluorescent products of oxidized hydroethidine (ethidium accumulation; red fluorescence) were increased 3 days after pKr-2 injection (Figure 5E,J) compared to PBS-injected control (Figure 5D,J). Treatment of IL-4Nab significantly reduced pKr-2-induced $\mathrm{O}_{2}{ }^{-}$ production in the CA1 layer of the hippocampus (Figure 5F,J).

To address the role of IL-4 on oxidative DNA damage, 8-OHdG levels were measured in pKr-2-injected CA1 layer of hippocampus in the presence or absence of IL-4Nab. Immunohistochemical analysis showed that $\mathrm{pKr}-2$ injection increased levels of 8-OHdG in the CA1 layer of hippocampus at 3 days after pKr-2 injection (Figure 5H,K), compared to PBS-injected control (Figure 5G,K). Treatment of IL-4Nab significantly reduced pkr-2-induced 8-OHdG levels in the CA1 layer of hippocampus (Figure 5I,K).

3.4. IL-4 Induces Oxidative/Nitrosative Stress in pKr-2-Injected CA1 Layer of Hippocampus In Vivo through $M P O$ and $i N O S$

Many studies demonstrated that myeloperoxidase (MPO) and inducible nitric oxide synthase (iNOS) participated in ROS/RNS production, resulting in neurodegeneration in vivo [1,4]. Moreover, microglia/macrophages and/or neutrophils were found to produce MPO- and iNOS-derived ROS/RNS in vivo [9,24-27]. Accordingly, we next investigated whether IL-4 could modulate expression of MPO and iNOS within reactive microglia/macrophages/neutrophils in the CA1 layer of hippocampus. Similar to our previous reports $[9,24]$, immunohistochemical analysis showed that expression of MPO and iNOS was significantly increased in OX-42 $2^{+}$microglia/macrophages/neutrophils at 3 days after pKr-2-injected CA1 layer of hippocampus in vivo (Figure 6B,E,K,L) compared to PBS-injected control (Figure $6 \mathrm{~A}, \mathrm{D}, \mathrm{K}, \mathrm{L}$ ). In contrast, treatment of IL-4Nab significantly reduced $\mathrm{pKr}$-2-induced MPO and iNOS levels in OX-42+ microglia/macrophages/neutrophils in the CA1 layer of hippocampus (Figure 6C,F,K,L). MPO expression is mainly localized in OX-42+ microglia/macrophages/neutrophils, but not in $\mathrm{GFAP}^{+}$astrocytes in pKr-2-injected CA1 layer of hippocampus in vivo (Figure 6J).

To assess the extent of ROS/RNS damages on proteins (protein nitration), we measured the levels of nitrotyrosine in the absence or presence of IL-4Nab. Immunohistochemical analysis revealed the significant increase in nitrotyrosine levels in the CA1 layer of hippocampus at 3 days after $\mathrm{pKr}-2$ injection (Figure 6H,M) compared to PBS-injected control (Figure 6G,M). Treatment with IL-4Nab attenuated levels of pKr-2-induced nitrotyrosine in the CA1 layer of hippocampus in vivo (Figure 6I,M).

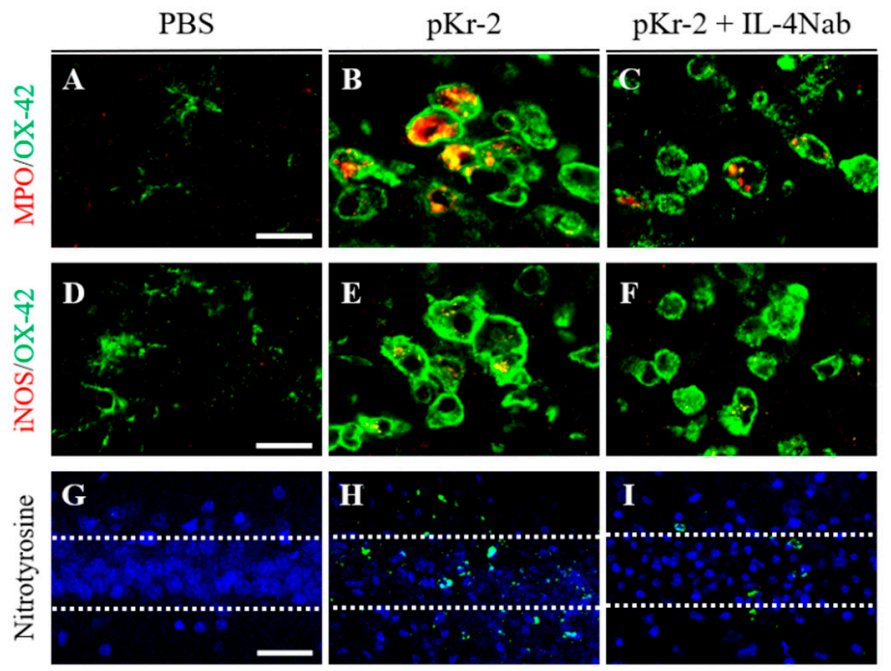

Figure 6. Cont. 
$\mathbf{J}$

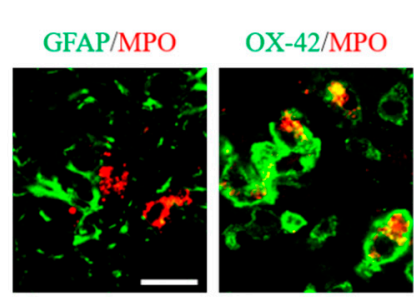

$\mathbf{L}$

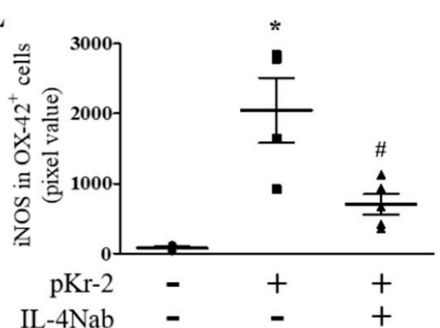

$\mathbf{K}$

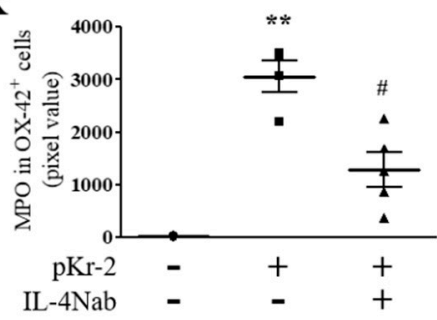

M

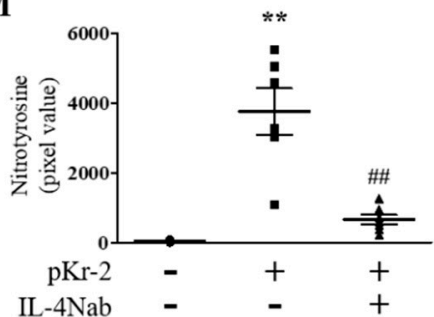

Figure 6. IL-4 modulates MPO- and iNOS-derived oxidative/nitrosative stress within activated microglia/macrophages in the CA1 layer of pKr-2-injected hippocampus in vivo. Sections (A,D,G (PBS); B,E,H (pKr-2); C,F,I (pKr-2 + IL-4Nab)) adjacent to those used in Figure 4 were processed for immunohistochemical staining. (A-C) Double immunofluorescence images of myeloperoxidase (MPO; red) and OX-42 (green) for microglia/macrophages/neutrophils and both images are merged (yellow) in the CA1 layer of hippocampus. Scale bar, $20 \mu \mathrm{m}$. (D-F) Double immunofluorescence images of inducible nitric oxide synthase (iNOS; red) and OX-42 (green) for microglia/macrophages/neutrophils and both images are merged (yellow) in the CA1 layer of hippocampus. Scale bar, $20 \mu \mathrm{m}$. (G-I) Immunofluorescence images of nitrotyrosine (green) to detect nitrosative damages in the CA1 layer of hippocampus. Nuclei were counterstained with DAPI (blue). Scale bar, $40 \mu \mathrm{m}$. (J) Double immunofluorescence images of GFAP (green, left panel) for astrocytes and MPO (red, left panel) or OX-42 (green, right panel) for microglia/macrophages/neutrophils and MPO (red, both panel) and both images are merged (yellow) in the CA1 layer of hippocampus. Scale bar, $20 \mu \mathrm{m}$. (K) Quantification of MPO expression in OX-42 ${ }^{+}$microglia/macrophages/neutrophils. ${ }^{* *} p<0.001$, significantly different from PBS (control). ${ }^{\#} p<0.01$, significantly different from $\mathrm{pKr}-2$. mean $\pm \mathrm{SEM} ; n=4$ to 5 in each group, ANOVA and Newman-Keuls analysis. (L) Quantification of iNOS expression in OX-42 ${ }^{+}$ microglia/macrophages/neutrophils. ${ }^{*} p<0.01$, significantly different from PBS (control). ${ }^{*} p<0.01$, significantly different from $\mathrm{pKr}-2$. mean $\pm \mathrm{SEM} ; n=4$ to 5 in each group, ANOVA and Newman-Keuls analysis. (M) Quantification of nitrotyrosine expression. ${ }^{* *} p<0.001$, significantly different from PBS (control). ${ }^{\# \#} p<0.001$, significantly different from $p K r-2$. mean \pm SEM; $n=4$ to 5 in each group, ANOVA and Newman-Keuls analysis. Dotted lines indicate the CA1 layer of the hippocampus.

\section{Discussion}

Oxidative/nitrosative stress contributes to increased levels of ROS/RNS formation that emerge as a crucial factor for neurodegeneration in vivo and in vitro $[1,2,4]$. iNOS is one of major source for excessive production of ROS/RNS, resulting in oxidative damage to proteins, lipids and DNA in AD [2,3]. Accumulating experimental evidence, including ours, showed that intracortical or intrahippocampal injection of thrombin, pKr-2, LPS and beta-amyloid 1-42 $_{2}$ increased the iNOS-derived ROS/RNS production in vivo $[9,21,28-30]$. The results of the present study demonstrate that neutralization of IL-4 significantly decreases $\mathrm{pKr}$-2-induced ROS production and oxidative damages on DNA and protein and prevents $\mathrm{pKr}$-2-induced degeneration of hippocampal neurons in vivo. This is similar to our previous studies showing that neutralization of IL-4 attenuated ROS production and prevented pKr-2-, thrombin- and LPS-induced degeneration of cortical neurons in vivo [9,18,22]. It is therefore likely that IL-4 might function as proinflammatory mediator in $\mathrm{pKr}$-2-lesioned hippocampus in vivo through production of iNOS-derived ROS/RNS. 
Accompanying microglial iNOS-derived ROS/RNS, several lines of evidence demonstrate that MPO-derived ROS/RNS can trigger neuronal death-related signaling cascades in vivo [4,31,32]. MPO is the key enzyme involved in the generation of cytotoxic ROS/RNS [32-34], resulting in oxidative damages to proteins, lipids and DNA in neurodegenerative disorders. Several reports, including ours, have demonstrated that various antioxidants, such as glutathione, ethyl pyruvate and capsaicin attenuated MPO-derived ROS/RNS levels and oxidative damages in vivo and in vitro [31,35,36]. Our results showed that accompanying reduction of ROS/RNS levels and oxidative damages, neutralization of IL-4 significantly attenuated MPO expression and neurodegeneration in $\mathrm{pKr}$-2-injected hippocampus in vivo. This indicates that like the iNOS, production of MPO-derived ROS/RNS might be regulated by IL-4. The present results have demonstrated that IL-4Nab partially, not fully attenuated oxidative stress markers such as iNOS/MPO. This may be a concentration effect as only one dose of IL-4Nab was used here, indicating that IL-4 is not the only factor to regulate iNOS/MPO under our current experimental conditions.

MPO is expressed in astrocytes, microglia/macrophages and neutrophil under neuropathological conditions $[4,25,31,32,35]$. In the present study, we demonstrate that MPO is colocalized with $\mathrm{OX}-42^{+}$microglia/macrophages, but not in $\mathrm{GFAP}^{+}$astrocytes in the CA1 layer of pKr-2-injected rat hippocampus invivo. This is inconsistent with previous reports showing that MPO was expressed in $\mathrm{GFAP}^{+}$astrocytes, but not in reactive microglia in the substantia nigra of 1-methyl-4-phenyl-1,2,3,6-tetrahydropyridine (MPTP) mice [26,31,35]. This discrepancy may reflect differences in toxins used (pKr-2 versus MPTP), regions studies (hippocampus versus substantia nigra) and/or animals (rat versus mouse) in each experiment. This interpretation is supported by previous findings that MPO is expressed in neutrophil, but not in astrocytes in mice exposed to spinal cord injury [37]. However, it cannot exclude the possibility that in pKr-2-injected hippocampus, MPO is expressed in neutrophil because of absence of distinguishable marker between microglia/macrophages and neutrophil.

Moreover, microglia are able to acquire either proinflammatory phase (M1) or anti-inflammatory phase (M2) during neuroinflammation $[38,39]$. In the present study, immunohistochemical analysis showed that IL-4Nab inhibited both microglia activation and iNOS/MPO expression within microglia. These results suggest that IL-4 might play a dual action such as microglial activation and/or polarization in $\mathrm{pKr}$-2-lesioned hippocampus in vivo.

\section{Conclusions}

In summary, the major finding of this manuscript is the observation that endogenous IL-4 expressed within reactive microglia/macrophages aggravates $\mathrm{pKr}$-2-induced neurodegeneration of the rat hippocampal CA1 area in vivo. This occurs through IL-4-induced oxidative stress since IL-4 neutralization reduces ROS/RNS production, number of $\mathrm{iNOS}^{+}$and $\mathrm{MPO}^{+}$reactive microglia/macrophages and oxidative damages, resulting in neuroprotection of pKr-2-treated hippocampus CA1 area in vivo. Thus, our results suggest that IL-4 contributes to the neurodegenerative processes in which neuroinflammation and oxidative stress are implicated.

Author Contributions: Conceptualization, Y.C.C. and B.K.J.; data curation, Y.C.C., J.Y.J. and B.K.J.; formal analysis, Y.C.C. and J.Y.J.; funding acquisition, Y.C.C. and B.K.J.; investigation, Y.C.C. and J.Y.J.; project administration, B.K.J.; supervision, B.K.J.; validation, Y.C.C. and J.Y.J.; writing-original draft, Y.C.C. and J.Y.J. and B.K.J. All authors have read and agreed to the published version of the manuscript.

Funding: This research was funded by the National Research Foundation of Korea (NRF) grant by the Korean Government NRF-2019R1A2C2007897, NRF-2019R1I1A1A01061729, NRF-2017M3C7A1031105 and NRF-2018R1A6A1A03025124.

Conflicts of Interest: The authors declare no conflict of interest. 


\section{References}

1. Cobb, C.A.; Cole, M.P. Oxidative and nitrative stress in neurodegeneration. Neurobiol. Dis. 2015, 84, 4-21. [CrossRef] [PubMed]

2. Dasuri, K.; Zhang, L.; Keller, J.N. Oxidative stress, neurodegeneration, and the balance of protein degradation and protein synthesis. Free. Radic. Biol. Med. 2013, 62, 170-185. [CrossRef]

3. Liochev, S.I. Reactive oxygen species and the free radical theory of aging. Free. Radic. Biol. Med. 2013, 60, 1-4. [CrossRef] [PubMed]

4. Chung, Y.C.; Ko, H.W.; Bok, E.; Park, E.S.; Huh, S.H.; Nam, J.H.; Jin, B.K. The role of neuroinflammation on the pathogenesis of Parkinson's disease. BMB Rep. 2010, 43, 225-232. [CrossRef] [PubMed]

5. Lin, M.T.; Beal, M.F. Mitochondrial dysfunction and oxidative stress in neurodegenerative diseases. Nat. Cell Biol. 2006, 443, 787-795. [CrossRef] [PubMed]

6. Hickman, S.; Izzy, S.; Sen, P.; Morsett, L.; El Khoury, J. Microglia in neurodegeneration. Nat. Neurosci. 2018, 21, 1359-1369. [CrossRef] [PubMed]

7. Wolf, S.A.; Boddeke, H.W.G.M.; Kettenmann, H. Microglia in Physiology and Disease. Annu. Rev. Physiol. 2017, 79, 619-643. [CrossRef]

8. Colonna, M.; Butovsky, O. Microglia Function in the Central Nervous System During Health and Neurodegeneration. Annu. Rev. Immunol. 2017, 35, 441-468. [CrossRef]

9. Jeong, J.Y.; Chung, Y.C.; Jin, B.K. Interleukin-4 and Interleukin-13 Exacerbate Neurotoxicity of Prothrombin Kringle-2 in Cortex In Vivo via Oxidative Stress. Int. J. Mol. Sci. 2019, 20, 1927. [CrossRef]

10. Kim, S.R.; Chung, E.S.; Bok, E.; Baik, H.H.; Chung, Y.C.; Won, S.Y.; Joe, E.; Kim, T.H.; Kim, S.S.; Jin, M.Y.; et al. Prothrombin kringle-2 induces death of mesencephalic dopaminergic neurons in vivo and in vitro via microglial activation. J. Neurosci. Res. 2009, 88, 1537-1548. [CrossRef]

11. Won, S.Y.; Choi, S.H.; Jin, B.K. Prothrombin kringle-2-induced oxidative stress contributes to the death of cortical neurons in vivo and in vitro: Role of microglial NADPH oxidase. J. Neuroimmunol. 2009, 214, 83-92. [CrossRef] [PubMed]

12. Shin, W.-H.; Jeon, M.-T.; Leem, E.; Won, S.-Y.; Jeong, K.H.; Park, S.-J.; McLean, C.; Lee, S.J.; Jin, B.K.; Jung, U.J.; et al. Induction of microglial toll-like receptor 4 by prothrombin kringle-2: A potential pathogenic mechanism in Parkinson's disease. Sci. Rep. 2015, 5, 14764. [CrossRef]

13. Berzin, T.M.; Zipser, B.D.; Rafii, M.S.; Kuo-Leblanc, V.; Yancopouloš, G.D.; Glass, D.J.; Fallon, J.R.; Stopa, E.G. Agrin and microvascular damage in Alzheimer's disease. Neurobiol. Aging 2000, 21, 349-355. [CrossRef]

14. Quarta, A.; Berneman, Z.; Ponsaerts, P. Neuroprotective modulation of microglia effector functions following priming with interleukin 4 and 13: Current limitations in understanding their mode-of-action. BrainBehav. Immun. 2020, 88, 856-866. [CrossRef]

15. Sholl-Franco, A.; Da Silva, A.G.L.S.; Adão-Novaes, J. Interleukin-4 as a Neuromodulatory Cytokine: Roles and signaling in the nervous system. Ann. N. Y. Acad. Sci. 2009, 1153, 65-75. [CrossRef]

16. Ravelli, K.G.; Santos, G.D.; Dos Santos, N.B.; Munhoz, C.D.; Azzi-Nogueira, D.; Campos, A.C.; Pagano, R.L.; Britto, L.R.; Hernandes, M.S. Nox2-dependent neuroinflammation in an EAE model of multiple sclerosis. Transl. Neurosci. 2019, 10,1-9. [CrossRef]

17. Yang, M.-S.; Park, E.J.; Sohn, S.; Kwon, H.J.; Shin, W.-H.; Pyo, H.K.; Jin, B.; Choi, K.S.; Jou, I.; Joe, E.-H. Interleukin-13 and -4 induce death of activated microglia. Glia 2002, 38, 273-280. [CrossRef]

18. Nam, J.H.; Park, K.W.; Park, E.S.; Lee, Y.B.; Lee, H.G.; Baik, H.H.; Kim, Y.S.; Maeng, S.; Park, J.; Jin, B.K. Interleukin-13/-4-induced oxidative stress contributes to death of hippocampal neurons in abeta1-42-treated hippocampus in vivo. Antioxid. Redox Signal 2012, 16, 1369-1383. [CrossRef]

19. Paxinos, G.; Watson, C. The Rat Brain in Stereotaxic Coordinates; Academic Press: San Diego, CA, USA, 1982.

20. Baek, J.Y.; Jeong, J.Y.; Kim, K.I.; Won, S.-Y.; Chung, Y.C.; Nam, J.H.; Cho, E.J.; Ahn, T.-B.; Bok, E.; Shin, W.-H.; et al. Inhibition of Microglia-Derived Oxidative Stress by Ciliary Neurotrophic Factor Protects Dopamine Neurons In Vivo from MPP+ Neurotoxicity. Int. J. Mol. Sci. 2018, 19, 3543. [CrossRef]

21. Choi, S.H.; Lee, D.Y.; Kim, S.U.; Jin, B.K. Thrombin-Induced Oxidative Stress Contributes to the Death of Hippocampal Neurons In Vivo: Role of Microglial NADPH Oxidase. J. Neurosci. 2005, 25, 4082-4090. [CrossRef] 
22. Park, K.W.; Baik, H.H.; Jin, B.K. Interleukin-4-induced oxidative stress via microglial NADPH oxidase contributes to the death of hippocampal neurons in vivo. Curr. Aging Sci. 2008, 1, 192-201. [CrossRef] [PubMed]

23. Bok, E.; Cho, E.J.; Chung, E.S.; Shin, W.-H.; Jin, B.K. Interleukin-4 Contributes to Degeneration of Dopamine Neurons in the Lipopolysaccharide-treated Substantia Nigra in vivo. Exp. Neurobiol. 2018, 27, 309-319. [CrossRef] [PubMed]

24. Bok, E.; Chung, Y.C.; Kim, K.S.; Baik, H.H.; Shin, W.H.; Jin, B.K. Modulation of M1/M2 polarization by capsaicin contributes to the survival of dopaminergic neurons in the lipopolysaccharide-lesioned substantia nigra in vivo. Exp. Mol. Med. 2018, 50,1-14. [CrossRef]

25. Ji, K.-A.; Yang, M.-S.; Jeong, H.-K.; Min, K.-J.; Kang, S.-H.; Jou, I.; Joe, E.-H. Resident microglia die and infiltrated neutrophils and monocytes become major inflammatory cells in lipopolysaccharide-injected brain. Glia 2007, 55, 1577-1588. [CrossRef]

26. Choi, D.K.; Pennathur, S.; Perier, C.; Tieu, K.; Teismann, P.; Wu, D.C.; Jackson-Lewis, V.; Vila, M.; Vonsattel, J.P.; Heinecke, J.W.; et al. Ablation of the inflammatory enzyme myeloperoxidase mitigates features of Parkinson's disease in mice. J. Neurosci. 2005, 25, 6594-65600. [CrossRef] [PubMed]

27. Tieu, K.; Ischiropoulos, H.; Przedborski, S. Nitric oxide and reactive oxygen species in Parkinson's disease. IUBMB Life 2003, 55, 329-335. [CrossRef] [PubMed]

28. Jana, M.; Palencia, C.A.; Pahan, K. Fibrillar amyloid-beta peptides activate microglia via TLR2: Implications for Alzheimer's disease. J. Immunol. 2008, 181, 7254-7262. [CrossRef]

29. Jang, J.-H.; Lee, S.H.; Jung, K.; Yoo, H.-R.; Park, G. Inhibitory Effects of Myricetin on Lipopolysaccharide-Induced Neuroinflammation. Brain Sci. 2020, 10, 32. [CrossRef]

30. Limón, I.D.; Diaz, A.; Mendieta, L.; Chamorro, G.; Espinosa, B.; Zenteno, E.; Guevara, J. Amyloid- $\beta 25-35$ impairs memory and increases NO in the temporal cortex of rats. Neurosci. Res. 2009, 63, 129-137. [CrossRef]

31. Huh, S.H.; Chung, Y.C.; Piao, Y.; Jin, M.Y.; Son, H.J.; Yoon, N.S.; Hong, J.Y.; Pak, Y.K.; Kim, Y.S.; Hong, J.K.; et al. Ethyl Pyruvate Rescues Nigrostriatal Dopaminergic Neurons by Regulating Glial Activation in a Mouse Model of Parkinson's Disease. J. Immunol. 2011, 187, 960-969. [CrossRef]

32. Marik, C.; Felts, P.A.; Bauer, J.; Lassmann, H.; Smith, K.J. Lesion genesis in a subset of patients with multiple sclerosis: A role for innate immunity? Brain 2007, 130, 2800-2815. [CrossRef] [PubMed]

33. Arnhold, J.; Flemmig, J. Human myeloperoxidase in innate and acquired immunity. Arch. Biochem. Biophys. 2010, 500, 92-106. [CrossRef]

34. Hampton, M.B.; Kettle, A.J.; Winterbourn, C.C. Inside the neutrophil phagosome: Oxidants, myeloperoxidase, and bacterial killing. Blood 1998, 92, 3007-3017. [CrossRef] [PubMed]

35. Chung, Y.C.; Baek, J.Y.; Kim, S.R.; Ko, H.W.; Bok, E.; Shin, W.-H.; Won, S.-Y.; Jin, B.K. Capsaicin prevents degeneration of dopamine neurons by inhibiting glial activation and oxidative stress in the MPTP model of Parkinson's disease. Exp. Mol. Med. 2017, 49, e298. [CrossRef] [PubMed]

36. Lu, N.; Ding, Y.; Tian, R.; Peng, Y. Inhibition of myeloperoxidase-mediated oxidative damage by nitrite in SH-SY5Y cells: Relevance to neuroprotection in neurodegenerative diseases. Eur. J. Pharm. 2016, 780, 142-147. [CrossRef]

37. Genovese, T.; Esposito, E.; Mazzon, E.; Di Paola, R.; Meli, R.; Caminiti, R.; Bramanti, P.; Fink, M.P.; Cuzzocrea, S. Beneficial effects of ethyl pyruvate in a mouse model of spinal cord injury. Shock 2009, 32, 217-227. [CrossRef]

38. Orihuela, R.; McPherson, C.A.; Harry, G.J. Microglial M1/M2 polarization and metabolic states. Br. J. Pharmacol. 2016, 173, 649-665. [CrossRef]

39. Yao, K.; Zu, H.-B. Microglial polarization: Novel therapeutic mechanism against Alzheimer's disease. Inflammopharmacology 2019, 28, 95-110. [CrossRef]

Publisher's Note: MDPI stays neutral with regard to jurisdictional claims in published maps and institutional affiliations. 